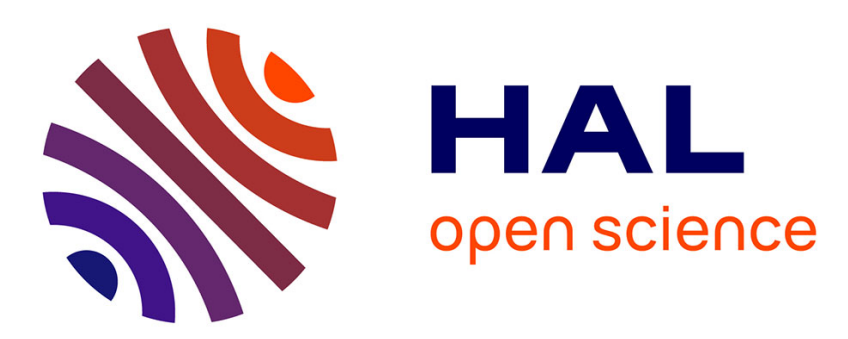

\title{
Le dispositif de gestion, moteur d'une dynamique entrepreneuriale collective. Le Projet Bio Loire Océan
}

Nathalie Schieb-Bienfait, Ivan Dufeu, Jean-Pierre Bréchet

\section{To cite this version:}

Nathalie Schieb-Bienfait, Ivan Dufeu, Jean-Pierre Bréchet. Le dispositif de gestion, moteur d'une dynamique entrepreneuriale collective. Le Projet Bio Loire Océan. Revue de l'Entrepreneuriat, A paraître, 19 (4), pp.57-79. 10.3917/entre.194.0057 . hal-02558960

\section{HAL Id: hal-02558960 \\ https://univ-angers.hal.science/hal-02558960}

Submitted on 25 Jun 2021

HAL is a multi-disciplinary open access archive for the deposit and dissemination of scientific research documents, whether they are published or not. The documents may come from teaching and research institutions in France or abroad, or from public or private research centers.
L'archive ouverte pluridisciplinaire HAL, est destinée au dépôt et à la diffusion de documents scientifiques de niveau recherche, publiés ou non, émanant des établissements d'enseignement et de recherche français ou étrangers, des laboratoires publics ou privés. 


\section{Le dispositif de gestion moteur d'une dynamique entrepreneuriale collective Le Projet Bio Loire Océan}

Pour citer cet article :

Schieb-Bienfait, N., Dufeu, I., \& Bréchet, J. P. (2020). Le dispositif de gestion, moteur d'une dynamique entrepreneuriale collective. Le Projet Bio Loire Océan. Revue de l'Entrepreneuriat, 19(4), 57-79. doi.org/10.3917/entre.194.0057

Résumé : Cette contribution porte sur les liens projet collectif - dispositif de gestion dans la dynamique entrepreneuriale qui a vu naître et se développer une association de producteurs de fruits et légumes biologiques. L'apport se veut principalement d'ordre théorique dans deux champs de recherche rapprochés : celui sur l'instrumentation gestionnaire, celui de la théorie de l'entreprise fondée sur le projet, théorie qui a déjà été envisagée et discutée dans le champ de l'entrepreneuriat et plus particulièrement dans le courant de l'émergence organisationnelle. La question abordée est la suivante: comment un collectif émerge, se constitue et se structure à travers ses dispositifs de gestion ? La recherche permet d'instruire finement le rôle des dispositifs dans le développement du projet entrepreneurial. L'instrumentation gestionnaire apparaît clairement au fondement de la possibilité de faire vivre dans la durée le projet collectif. Cette recherche apporte aussi une meilleure compréhension des processus relatifs à la découverte et à l'exploitation de l'opportunité entrepreneuriale dans le monde agricole, notamment dans la mouvance dite alternative.

Mots clés : projet, collectif, dispositif, régulation, entrepreneuriat 


\section{Introduction}

L'instrumentation de gestion a reçu moins d'attention de la part des chercheurs dans les contextes inter-organisationnels qu'à l'échelle de l'entreprise et de ses dispositifs internes. De même, les démarches entrepreneuriales, si elles n'ont pas été oubliées du point de vue des outils d'accompagnement, n'ont pas été réellement abordées pour instruire la dynamique «projet entrepreneurial - dispositif » dans l'esprit de la recherche sur les outils de gestion. Enfin, nous faisons le constat de la rareté des recherches sur les projets entrepreneuriaux dans l'univers agricole, et notamment lorsque le projet est initié par un collectif de producteurs concurrents sur le marché, en dehors du modèle coopératif bien connu.

Cette contribution engage les trois aspects dont nous venons de souligner le relatif délaissement dans l'univers de la recherche. Nous allons nous intéresser aux liens «projet collectif - dispositif de gestion »dans la dynamique entrepreneuriale qui a vu naître et se développer l'association de producteurs en agriculture biologique Bio Loire Océan (BLO). Cette association regroupe une cinquantaine de maraîchers et d'arboriculteurs sur le territoire de la région Pays de la Loire. Elle se donne pour mission de définir et d'animer un projet partagé, fortement exigeant sur le plan de l'engagement dans ce que nous pouvons désigner comme l'agriculture biologique équitable (Dufeu, Duchaine, Mc Namara et Morvan, 2016 ; Le Velly, Dufeu et Le Grel, 2016). Il s'agit d'un projet collectif affirmé sur le plan des principes et des valeurs, en même temps qu'original sur celui de l'intermédiation commerciale qu'il assure entre les offres du collectif de producteurs et la demande qu'ils veulent satisfaire. Le projet BLO offre ainsi des perspectives de réflexion et de diffusion intéressantes, à l'heure où le recours au local dans les approvisionnements alimentaires apparaît comme un réel enjeu pour nombre d'acteurs publics et privés. L'originalité de cette expérience va nous conduire à en privilégier une restitution fine que rend possible un accompagnement sur plusieurs années par un collectif de chercheurs.

La démarche d'élaboration du projet BLO au fil du temps va donc être examinée ici sous l'angle du rôle joué par les dispositifs de gestion, notamment de ceux qui permettent d'affirmer le projet collectif et de le faire vivre. Notre apport se veut principalement d'ordre théorique avec le souci de rapprocher le champ de recherche sur l'instrumentation gestionnaire et la théorie de l'entreprise fondée sur le $\operatorname{Projet}^{1}$ (TEFP). Cette dernière, déjà discutée dans le champ de l'entrepreneuriat ${ }^{2}$, met le Projet au fondement de l'action collective. Avec cette entrée par la TEFP, se pose pleinement la question de l'émergence de l'action collective et de sa construction, ce qui a été théorisé comme le passage du projet d'entreprendre au projet d'entreprise (Bréchet, 1994). De plus, ces deux univers théoriques - la TEFP et l'instrumentation gestionnaire - sont ici rapprochés dans le cadre englobant de la Théorie de la régulation sociale (TRS) de J.-D. Reynaud (1997).

Il est intéressant de pointer aussi l'enjeu d'une meilleure compréhension des réalités entrepreneuriales dans l'univers agricole (McElwee, 2006, 2008 ; Cook et Plunkett, 2006 ; Emin et Schieb-Bienfait, 2013), notamment celui des pratiques qui se veulent alternatives (Le Velly et Dufeu, 2016). Nous considérons enfin qu'un apport se joue dans les débats portant sur l'opportunité entrepreneuriale (Ngigol, 2004, 2015; Shane, 2003 ; Chabaud et Ngijol, 2004 ; Chabaud et Messeghem, 2010), du fait de l'inscription de la réflexion dans celle portant sur la construction du projet entrepreneurial.

\footnotetext{
${ }^{1}$ Nous convenons de mettre une majuscule au terme de projet lorsqu'il s'agit clairement de désigner le projet collectif et non seulement de projet au sens de l'expression de « management de projet ».

${ }^{2}$ Cf. numéro spécial de la Revue de l'entrepreneuriat, 2011, vol.10, avec notamment les articles de Asquin, Condor et Schmitt; Boutinet et Raveleau ; Bréchet et Schieb-Bienfait. Une lecture en termes de projet est également défendue par des auteurs majeurs en entrepreneuriat (Gartner, 1985, 1995 ; Steyaert, 2007 ; Hjorth et Johannisson, 2009).
} 
Notre question de recherche peut se formuler ainsi : comment un collectif émerge, se constitue et se structure à travers une instrumentation évolutive. Cette formulation générique se précise dans les trois questions suivantes qui organiseront notre réflexion :

1/ Comment le dispositif de gestion rend-il possible le projet collectif dans la phase d'émergence?

2/ Comment le dispositif accompagne-t-il le développement du projet collectif dans la phase de développement?

3/ Comment le dispositif fait-il évoluer le projet collectif au fil du temps ?

Nous construisons le propos en exposant dans un premier temps les choix théoriques et conceptuels retenus afin d'étudier les liens projet collectif - dispositifs gestionnaires (1). Dans un deuxième temps nous présenterons l'expérience BLO (2), avant de préciser, dans un troisième, les liens Projet-dispositifs et leur évolution au fil du temps (3). Nous terminerons par les analyses et les enseignements tirés de notre investigation. A la question de savoir si le dispositif de gestion peut être le moteur d'une dynamique entrepreneuriale collective, nous répondons par l'affirmative en précisant les interactions entre le projet collectif et les dispositifs qui l'instrumentent dans son émergence et son développement (4).

\section{Le Projet et ses dispositifs : un éclairage théorique}

Le concept de projet, au sens de projet collectif, se définit comme effort d'intelligibilité et de construction de l'action fondée sur l'anticipation (Desreumaux et Bréchet, 2009). En tant qu'effort d'intelligibilité, il contribue à la construction des savoirs requis pour entreprendre. En tant qu'effort de construction de l'action, il participe des régulations effectives de l'action collective. Le concept de projet ainsi compris apparaît comme indispensable pour penser l'acte entrepreneurial (Bréchet, Schieb-Bienfait, Desreumaux, 2009).

Que l'on dise que l'action collective engage un Projet ne doit pas surprendre. La convergence des intérêts individuels ne suffit pas pour comprendre l'action collective, pas plus que les valeurs ou les cadres interprétatifs. Il faut comprendre l'instauration d'une régulation. Le Projet participe de la création des règles et des régulations de l'action, ce que retient la Théorie de la régulation sociale (TRS) de J.-D. Reynaud ${ }^{3}$. On peut dire avec cet auteur que l'action collective naît d'un Projet par lequel l'acteur social se constitue en se dotant de règles singulières dans leur conception et dans leur pratique. Le consentement à faire vivre les règles, c'est-à-dire une régulation constitutive et régulatrice de l'action collective, fonde une possibilité d'action commune. S'il ne semble pas usurpé de parler d'une «communauté de projet» pour définir l'action collective (Reynaud, 2008), celle-ci doit être comprise comme communauté de règles vécues.

Observer un projet collectif, s'intéresser à son émergence et à sa vie, ce n'est pas négliger les valeurs et les principes dont sont porteurs les acteurs de l'action, mais c'est prioritairement s'intéresser aux régulations et aux règles engagées dans l'action. Bien entendu, les raisons d'agir des acteurs n'ignorent pas les contingences de l'action, les arbitrages et les compromis que parfois l'action nécessite. L'action a ses exigences de résultat et connaît ses épreuves. Pointer l'importance des attachements des protagonistes de l'action à certaines valeurs, comme c'est le cas chez BLO à propos du «bio-équitable », n'exclut pas non plus la prise en compte des intérêts qui sont parfaitement légitimes. Enfin, on ne peut envisager les régulations et les règles sans faire toute leur place aux dispositifs et outils de gestion. Puisque nous nous intéressons au projet collectif, il s'agit donc de porter notre attention sur les dispositifs et outils par lesquels le projet collectif existe et s'affirme.

Depuis les premiers travaux engagés par Berry (1983), l'approche par les outils de gestion est désormais bien inscrite dans le champ managérial (Cf. par exemple : Hatchuel et Moisdon, 1993 ; Moisdon, 1997 ; David, 1998 ; Aggeri et Labatut, 2010 ; De Vaujany, 2005,

\footnotetext{
${ }^{3}$ Cf. par exemple Reynaud (1997) ou pour une synthèse Bréchet (2008).
} 
2006; Chiapello et Gibert, 2013). Mais si la reconnaissance de l'omniprésence de l'instrumentation gestionnaire est partagée par la communauté scientifique en sciences de gestion, son étude en contexte entrepreneurial demeure encore discrète. C'est notamment le cas en phase d'émergence organisationnelle et de structuration d'un collectif d'acteurs. Le contexte qui est le nôtre d'un projet entre des producteurs indépendants, donc d'un projet que l'on pourrait qualifier d'inter-organisationnel, renforce ce constat. Le lien que nous cherchons à établir entre la formation du projet collectif et ses dispositifs relève encore d'une démarche exploratoire.

L'intérêt porté à l'instrumentation gestionnaire a donné lieu à une diversité de dénominations. A la suite de Aggeri et Labatut (2010), qui offrent une synthèse des diverses compréhensions plus ou moins larges ou restrictives (modèles, instruments, outils...) nous avons choisi de privilégier l'expression « dispositif de gestion » qui fait référence à un niveau d'analyse moins élémentaire que celui de l'outil ou de l'instrument. Le dispositif permet d'orienter le regard à la fois sur les outils de gestion et les acteurs de ces outils.

Ainsi l'action collective engage-t-elle toujours des dispositifs de gestion que l'on peut comprendre comme des « arrangements dans le temps et dans l'espace des personnes et des choses » pour reprendre les termes de Moisdon (2005 : 239). Et ces dispositifs se conçoivent, s'aménagent et se transforment en vue de finalités (Aggeri et Labatut, 2010). Dans la perspective de la TEFP, l'activité entrepreneuriale peut être étudiée comme une activité d'ingénierie, c'est-à-dire une activité de conception et de mise en œuvre de dispositifs qui structurent et rendent possible le projet collectif et donc l'action collective. Dans les termes de la TRS, on peut dire que les auteurs et acteurs du projet collectif effectuent un travail de régulation ${ }^{4}$ supporté par des dispositifs. Ce travail de régulation va rencontrer les contingences de l'action entrepreneuriale : il s'agit bien d'un effort toujours à reprendre pour construire les savoirs et les relations qui rendent l'action collective possible (Hatchuel, 2000). Mais de quelle grammaire dispose-t-on pour aborder ce travail instrumenté de régulation visant à faire vivre le projet collectif?

Nous poserons, pour finir sur cette mise au point théorique, que l'effort d'intelligibilité et de construction de l'action, avec ses dispositifs qui instrumentent la régulation, porte sur trois dimensions de tout projet collectif : la dimension éthico-politique, la dimension technicoéconomique, la dimension organisationnelle (Bréchet, 1994, Desreumaux et Bréchet, 2009). Dit autrement, tout projet collectif met en jeu : 1/ des dimensions éthiques et politiques, exprimées ou simplement vécues ; $2 /$ des orientations et des choix technoéconomiques portant sur les produits et services proposés en même temps que les compétences requises ; 3/ des modalités organisationnelles de mise en ouvre qui sont autant de pratiques anticipées. Ces trois dimensions vont guider notre investigation théorique et empirique dans la restitution de la vie du Projet BLO. Dans cette perspective, la vie du projet collectif recouvre plusieurs dispositifs : il s'agit notamment de la charte et du cahier des charges pour ce qui est de la dimension éthicopolitique; des outils de l'intermédiation marchande pour la dimension économique; et des aspects de mise en œuvre prévus pour la dimension organisationnelle.

\section{Encadré 1 : Repères sur la méthodologie}

Dans le cadre d'une recherche longitudinale, nous avons étudié l'évolution du projet entrepreneurial de BLO sur près de 10 années, avec - depuis cinq ans - le déploiement d'une démarche de recherche-accompagnement (Bréchet, Emin, Schieb-Bienfait, 2014) auprès de l'équipe de pilotage et d'animation de BLO. C'est dans cette perspective temporelle longue que nous avons choisi d'explorer les dispositifs de gestion déployés lors de la genèse et de la vie de ce projet entrepreneurial collectif, à partir d'une analyse de données primaires et secondaires,

\footnotetext{
${ }^{4}$ Nous n'intégrons pas ici la problématique de l'autonomie et du contrôle et donc nous n'opérons pas de distinction entre la régulation dite de contrôle et celle dite d'autonomie.
} 
d'entretiens, de participations à des réunions de travail et des comités (assemblée générale conseil d'administration, comité technique, comité de pilotage, groupes projet...).

\section{De l'émergence à l'affirmation du Projet Bio Loire Océan}

Dans un contexte de développement de la demande de produits biologiques et locaux et de sollicitation croissante en provenance de la grande distribution, quelques producteurs biologiques des Pays de la Loire décident en 1995 de se concerter sur la stratégie commerciale à adopter. Autour d'un café au Marché d'Intérêt National (MIN) de Nantes, ils prennent conscience de la nécessité de coordonner leurs offres pour répondre aux attentes des marchés tout en évitant de dépendre d'eux. Ceci devait les conduire à ce qui allait devenir un projet associatif original dont nous allons retracer les principales étapes.

\subsection{La naissance du projet associatif}

A sa création en 1997, l'association BLO se définit dans ces termes : «Bio Loire Océan est une association de producteurs dont l'objectif est de développer et de structurer la filière des fruits et légumes uniquement biologiques. » (extrait des statuts de l'association). Dans ce texte fondateur de 1997, l'intérêt de s'organiser face à la demande croissante des grandes surfaces en fruits et légumes biologiques est formulé en quatre points : (1) « être présent ensemble et avec le même langage [...] afin d'orienter le marché des GMS (Grandes et Moyennes Surfaces de vente) vers le respect des principes auxquels nous sommes attachés ; (2) se regrouper pour être un interlocuteur de poids devant les clients tels que les GMS ; (3) se concerter sur les gammes de produits, les volumes et surtout les prix ; (4) mettre en commun des expériences tant techniques que commerciales ».

Ces objectifs évoquent ceux que peuvent se donner certaines coopératives agricoles. Mais le choix d'une forme associative, plutôt que coopérative, n'est pas le fruit du hasard. Les fondateurs sont en effet majoritairement des déçus des coopératives agricoles et ne souhaitent surtout pas y retourner. Ils ont une réelle défiance vis-à-vis de ces formes d'organisation où, selon eux, le pouvoir de décision finit par échapper aux producteurs pour revenir aux responsables de la coopérative qui privilégient des approches marketing et commerciales traditionnelles : «C'est dû au mode de gestion du système coopératif qui est trop contraignant. Aujourd'hui on privilégie le client et pas le producteur, même si c'est en théorie géré par des producteurs. En pratique, les managers de la coop ont beaucoup de pouvoir. Quand il y a des efforts à faire c'est toujours le producteur qui doit les faire. [...] Beaucoup des commerciaux de ces coopératives sont rémunérés par rapport aux volumes vendus et pas par rapport à ce qu'il reste au producteur. Tant que ça restera sur ces bases-là, ils ne s'en sortiront pas. Et personne ne veut faire changer le système » (direction, 2012).

Les producteurs veulent donc à l'époque une structure simple et souple, qui privilégie l'implication des acteurs producteurs pour maitriser à la fois le Projet dans ses dimensions éthico-politique et technico-économiques. Ils affirment toujours actuellement cette volonté de garder la main sur la structure. La forme associative permet également aux associés de conserver une certaine liberté vis-à-vis de l'organisation. Le responsable synthétise ainsi ces idées: "Bio Loire Océan s'est distinguée très rapidement par la volonté des producteurs de mettre en place sur la Région un outil au service des producteurs. L'association a donc choisi d'innover en mettant en place une structure légère et peu coûteuse (un animateur) et d'associer fortement les producteurs à la mise en place des actions » (direction, 2012). 
Coopérer tout en restant indépendants, voilà donc leur désir. Le projet politique est clair, le projet économique est posé et l'organisation est supposée les servir. Mais les débuts seront difficiles, malgré un contexte globalement favorable ${ }^{5}$.

\subsection{L'émergence organisationnelle}

Les premières actions concrètes datent de 1998. Pour répondre à la sollicitation de Carrefour, un grossiste du MIN - Biosélection - propose à BLO d'assurer le regroupement des produits agricoles de ses adhérents, de les conditionner et de les livrer en GMS. Mais les difficultés arrivent très rapidement avec le dépôt de bilan du grossiste qui « laisse une ardoise » à certains producteurs. Cet épisode, vécu douloureusement par les membres de l'association, renforce chez certains des visions individualistes et érode la volonté d'action collective. Nous sommes en 1999, BLO a neuf adhérents. L'association qui vivote ne s'est pas encore dotée de dispositifs de gestion. Le projet associatif peine à s'affirmer.

En 2000, un évènement déclencheur réactive l'association. La société coopérative Biocoop, qui se développe, exprime la demande de regroupement de l'offre des producteurs. «A partir de ce moment-là, on essaye de mettre en place une plateforme de centralisation et en même temps de conserver l'indépendance des producteurs qui sont habitués à tout gérer chez eux » (vice-président, 2012). Cette demande permet alors à un noyau de six producteurs de faire preuve d'initiative et de « reprendre la main » avec la volonté de faire à nouveau quelque chose ensemble. L'embauche par l'association d'une chargée de mission à temps plein au milieu de l'année 2000, donne également une nouvelle impulsion, notamment pour ce qui est de la structuration des dispositifs de gestion. Elle permet d'entamer un travail de planification avec Biocoop et d'engager un suivi technique de la production. Sa démission en 2002 sera un nouveau coup dur.

BLO va toutefois redémarrer rapidement, courant 2003, avec l'arrivée de la nouvelle et actuelle - coordinatrice mise à disposition à temps partiel par le GAB (Groupement d'Agriculteurs Biologiques) Anjou. Sa mission se centre davantage sur le travail de structuration et d'animation de la filière fruits et légumes pour répondre aux demandes réitérées du réseau Biocoop. Dans un contexte de développement des tonnages et d'élargissement de la gamme en fruits et légumes, une planification renforcée des approvisionnements sur sa plateforme logistique de Rennes est indispensable à Biocoop. Dans ces circonstances, l'assemblée générale (AG) de 2004 va constituer un tournant définitif. Le bureau composé de quelques agriculteurs très investis et de la coordinatrice, rédige un bilan et un calendrier d'actions pour la période 2004-2006. Le document de six pages prévoit notamment les points de regroupement logistique, l'harmonisation sur les prix, l'amélioration de la planification de l'offre, une réflexion sur un système de facturation commune, le lancement du logo BLO, la rédaction du cahier des charges. La plupart des projets évoqués dans ce document voient le jour dans les cinq années qui suivent. La facturation commune, la planification et l'offre mutualisées se mettent en place progressivement à partir de 2005, essentiellement à destination du réseau Biocoop. Ces activités font appel à des outils de plus en plus évolués, qui reposent sur des plateformes en ligne à partir de 2009. Les offres des membres de BLO sont visibles de tous chaque jour, et les achats et les prix de Biocoop sont renseignés en temps réel. Nous verrons plus tard que ces outils de gestion sont plus innovants qu'il n'y parait, à l'image du projet associatif lui-même, dans son ensemble.

Entre 2005 et 2010, le chiffre d'affaires avec les quatre plateformes du réseau Biocoop va croître d'environ 50\% par an, alors que le nombre d'adhérents augmente lentement. Chemin faisant, l'association a su en même temps créer de nouvelles missions et répondre à d'autres types d'attentes pour les adhérents «Historiquement, tout le schéma d'organisation, de fonctionnement, on l'a mis en place avec les producteurs pour fournir Biocoop. Mais l'objectif de BLO n'était pas que de répondre à Biocoop, c'était quand même aussi de s'organiser et de

\footnotetext{
${ }^{5}$ L'association ne s'est pas encore dotée d'instruments de dispositifs de gestion à cette époque.
} 
structurer plus largement la filière. Ce qu'on observe maintenant, c'est l'adhésion de nos producteurs à différents services, aux différentes missions qui ont été développées ces dernières années » (administrateur, 2016).

\subsection{Le projet collectif s'affirme}

Aujourd'hui, BLO est la première association de producteurs de fruits et légumes biologiques sur la Région, avec presque 70 adhérents, 260 salariés en équivalents temps-pleins, pour plus de 1/3 de la production ligérienne de fruits et légumes. «En termes de surface, ça représente près de 300 hectares de maraîchage et un peu plus de 200 hectares de vergers, pour un chiffre d'affaires cumulé des adhérents de BLO de 18 millions d'euros» (animatrice 2015). En moyenne, $20 \%$ des productions des adhérents sont commercialisés par l'intermédiaire de l'association. Ce pourcentage peut être variable, mais l'association veille à ce qu'il ne soit pas trop important pour que les producteurs développent leurs propres marchés « afin d'assurer leur indépendance vis-à-vis du système ». Mentionnons aussi le fait que $10 \%$ des adhérents ne font pas de commerce via l'association ; certains viennent y chercher avant tout les échanges avec des pairs, la participation à des programmes particuliers, comme par exemple le programme d'action sur les semences paysannes. En 2017, les ressources financières de l'association sont les suivantes : 1) environ $60 \%$ proviennent des cotisations des adhérents (un fixe très faible) et du prélèvement de $3 \%$ du chiffre d'affaires réalisé via $\mathrm{BLO})$; 2) Pour environ $40 \%$, les ressources proviennent de contrats de recherche (Fondation de France, Région etc...) et de subventions de l'État et de la Région. Les ventes qui transitent par BLO, sur lesquelles il y a donc une retenue, sont pour près de $80 \%$ à destination du réseau Biocoop, le reste étant majoritairement lié à l'association de distribution paniers de légumes dans le cadre des «Paniers Bio Solidaires ».

$\mathrm{La}$ gouvernance de BLO répond au principe «un producteur, une voix ». Les producteurs sont les seuls à prendre les décisions. Le conseil d'administration est composé d'une dizaine de membres et le taux de participation aux AG est de 60 à $80 \%$ selon les années. Pour ce qui est des projets portés au quotidien, BLO étant une association, les producteurs sont impliqués dans la mesure où ils le souhaitent. C'est d'ailleurs un vœu de l'association qu'elle soit mue par les projets de ses adhérents. Certains acteurs-clés sont très présents et mobilisés, mais la diversité des activités portées par BLO (recherche et expérimentation agronomiques, développement de systèmes de paniers, restauration collective...) est telle que les agriculteurs se retrouvent presque tous impliqués dans des activités collectives. Les acteurs de BLO sont aussi «force de proposition pour de nouvelles actions collectives » jamais exclues a priori, dès lors qu'elles sont en phase avec le projet d'ensemble. L'état d'esprit de l'association s'est affirmé : «Bio Loire Océan n'est pas un bureau de vente. Ce n'est pas la mission de l'association. Par contre, les agriculteurs qui veulent intégrer un collectif pour pouvoir échanger avec d'autres producteurs, pour pouvoir réfléchir sur les circuits de commercialisation et diversifier leurs commercialisations, comme participer aux actions qui sont mises en place sur la restauration collective ou sur le programme expérimental en semences paysannes, là il n'y a pas de problème. On va être capable de les accompagner sur ces thématiques » (coordinatrice, 2016). Le travail sur les textes fondateurs (charte commune, cahier des charges) par exemple, s'est effectué avec cette philosophie (Dufeu, Le Velly, Bréchet et Loconto, 2020).

L'équipe opérationnelle de BLO s'est quant à elle largement étoffée, et ce sont désormais cinq salariés qui sont chargés de différentes missions, en lien avec les projets en cours. L'accompagnement et le développement de la commercialisation demeurent l'activité première de l'association. Reconnue comme OEPB (Organisation Economique de Producteurs Biologiques), BLO participe également aux discussions nationales et à la structuration de la filière. 


\section{Les dispositifs de gestion dans l'évolution du projet entrepreneurial collectif}

L'évolution des dispositifs de gestion, simplement évoqués jusqu'ici, accompagne celle $\mathrm{du}$ projet collectif entrepreneurial. Les interactions acteurs-outils, sur lesquelles s'appuie le travail de régulation des acteurs de BLO, témoignent des liens Projet-dispositif qui sont au cœur de l'action collective.

\subsection{Les dispositifs d'accompagnement du travail sur le Projet}

Faire coopérer des acteurs agricoles, concurrents et souvent soucieux de maintenir leur indépendance n'est pas aisé. Si l'initiative associative a été suscitée par la conjonction de plusieurs facteurs à la fois favorables et défavorables que nous avons évoqués, l'affirmation du projet au fil du temps a nécessité des discussions, des négociations pour faire évoluer le projet dans son expression et dans ses pratiques.

Les agriculteurs adhérents qui constatent la méconnaissance respective de leurs activités et leur relative incapacité à informer les tiers comme Biocoop sur l'offre, que ce soit en termes de volumes ou de tarifs, mesurent qu'il leur est nécessaire de s'organiser. La mise en place de dispositifs s'impose pour leur permettre d'échanger afin de capitaliser sur leurs expériences et de travailler sur leur Projet. L'objectif est de mieux se connaître, de s'accorder sur le périmètre de leurs activités communes, et précisément sur les termes de la convention mise en place avec Biocoop. Ainsi, au travail initial sur le Projet, fait suite un travail plus poussé sur les textes fondateurs en même temps que sont revues les missions du bureau et du CA pour affirmer une gouvernance associative exigeante (Tableau 1 ).

Tableau 1 : Les dispositifs d'accompagnement du travail sur le Projet

\begin{tabular}{|c|c|c|}
\hline $\begin{array}{c}\text { Dispositif } \\
\text { acteurs - outils }\end{array}$ & Finalités & Contribution à l'élaboration du Projet \\
\hline $\begin{array}{ll}\text { Le travail initial sur le } \\
\text { projet associatif } \\
-\quad \begin{array}{l}\text { Rencontres } \\
\text { régulières entre }\end{array} \\
\text { producteurs } \\
\text { - } \quad \text { Comité technique } \\
\text { - Conseil } \\
\text { d'administration } \\
\text { - } \quad \text { Bureau, dont } \\
\text { coordinatrice }\end{array}$ & $\begin{array}{ll}\text { - } & \text { Etre partie prenante de la } \\
\text { structuration de la bio en région Pays } \\
\text { de la Loire. } \\
\text { - }\end{array}$ & $\begin{array}{ll}\text { - } & \text { meilleure connaissance des acteurs, des } \\
\text { outils de production, des volumes via } \\
\text { des processus d'échanges d'informations } \\
\text { participation de tous pour faire remonter } \\
\text { l'information et échanger avec les } \\
\text { producteurs-collègues de l'association. } \\
\text { - } \quad \text { apprentissages croisés entre producteurs } \\
\text { - } \\
\text { développement de la confiance entre } \\
\text { acteurs et redéfinition des finalités du } \\
\text { Projet }\end{array}$ \\
\hline $\begin{array}{l}\text { Le travail sur les textes } \\
\text { fondateurs } \\
\text { - } \quad \text { La charte } \\
\text { - } \quad \text { Le cahier des } \\
\quad \text { charges } \\
\text { - } \quad \text { La marque }\end{array}$ & 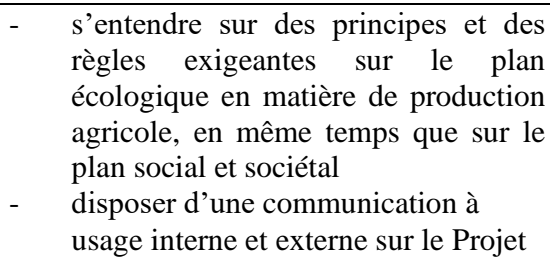 & 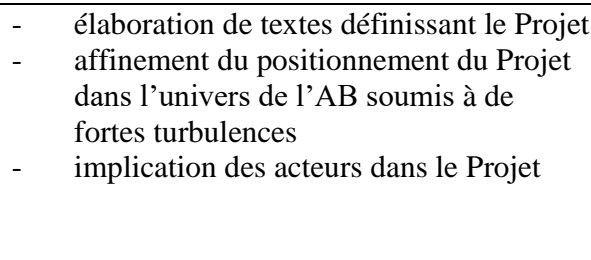 \\
\hline $\begin{array}{l}\text { La gouvernance } \\
\text { associative }\end{array}$ & $\begin{array}{ll}\text { - } & \text { se doter d'un processus de décision } \\
\text { favorisant la collégialité } \\
\text { - } \\
\text { s'assurer de l'intégration réussie de } \\
\text { nouveaux membres }\end{array}$ & $\begin{array}{l}\text { affirmation des principes et des valeurs } \\
\text { du Projet et suivi de leur prise en compte } \\
\text { dans l'action }\end{array}$ \\
\hline
\end{tabular}

Les producteurs, qui se découvrent mutuellement, constatent qu'ensemble ils peuvent peser sur les régulations marchandes, au bénéfice du collectif. Ils prennent alors confiance dans leur projet initial. Progressivement, l'affirmation et l'ancrage de leur association dans le paysage agricole ligérien vont les conduire à réinterroger le contenu même de leur projet commun, aussi bien dans ses finalités que dans ses modalités. La pratique de la concertation s'instaure dans leurs instances représentatives - bureau et $\mathrm{CA}$, aussi bien que dans la vie collective au quotidien, 
avec un rôle essentiel joué par la cellule de coordination. Ces pratiques exigeantes permettent l'affirmation de nouvelles ambitions pour le projet politique et celles-ci vont s'exprimer dans la charte commune et l'élaboration d'un cahier des charges spécifique (Dufeu, Le Velly, Bréchet et Loconto, 2020).

La charte a été rédigée une première fois en 2005 et renouvelée en 2013. Elle s'adresse principalement aux adhérents. Elle concrétise un engagement pour des principes et des objectifs communs partagés par le groupe. Outre la non-utilisation de produits chimiques de synthèse dans l'exercice de leur métier, les producteurs se veulent attentifs au développement et à la pérennité des outils de production présents sur le territoire des Pays de la Loire. Ce document de deux pages constitue le texte fondateur qui unit les adhérents en précisant le Projet de l'association pour une production de fruits et légumes biologiques cohérente et solidaire, en prise avec les territoires. Cette charte est évolutive, elle sert de fil conducteur à l'élaboration du cahier des charges et de ses règles de certification qui complètent le règlement européen de l'agriculture biologique. Le cahier des charges est donc un document beaucoup plus long et opérationnel qui traduit les principes généraux en critères observables. Les principales rubriques sont : biodiversité cultivée et sauvage, respect de la saisonnalité, protection des cultures, usage de l'eau, lien au sol, vie des sols et fertilisation, pour finir par trois chapitres sur les pratiques socioéconomiques.

Suite à ce travail poussé sur la charte et sur le cahier des charges, dans la perspective d'en contrôler l'application et le respect, la direction de BLO décide de faire connaître ces textes fondateurs aux parties prenantes externes. C'est en s'appuyant sur la marque collective, la marque «Bio Loire Océan », déposée en 2001, que cette démarche se réalise. D’abord peu utilisée, la marque a été « ressortie des placards » en 2010 et centrée davantage sur le logo «Bio Loire Océan ». Ce qui a évolué également, c'est le rôle qui lui est attribué. Il y a désormais une véritable prise de conscience de l'importance de capitaliser sur la marque «pour donner au collectif une bonne visibilité nationale et une notoriété intéressante» (coordinatrice, 2016). Aujourd'hui, ce dispositif à trois niveaux est donc opérationnel : la charte pour édicter les principes généraux ; le cahier des charges pour opérationnaliser les principes ; la marque pour valoriser ces principes et ces règles.

3.2. L'expérimentation des premiers dispositifs d'intermédiation marchande dans la phase d'émergence organisationnelle

Dans la phase que l'on peut considérer comme celle de l'émergence organisationnelle, des problématiques d'organisation et de renforcement de la coopération sont abordées de manière récurrente lors des AG et des CA. BLO expérimente différents dispositifs. Les préoccupations ont trait principalement aux problématiques de regroupement de l'offre des adhérents et aux expéditions - non pas pour livrer des magasins, mais pour livrer des plateformes Biocoop, qui ensuite approvisionnent les magasins de leur réseau. Ces premières années sont marquées par les préoccupations d'expédition pour servir ce grossiste (Tableau 2). 
Tableau 2 : Les premiers dispositifs d'intermédiation marchande

\begin{tabular}{|l|l|l|}
\hline \multicolumn{1}{|c|}{$\begin{array}{l}\text { Dispositif } \\
\text { acteurs - outils }\end{array}$} & \multicolumn{1}{|c|}{ Finalités } & Contributions à l'élaboration du Projet \\
\hline$-\begin{array}{l}\text { réunions } \\
\text { appels } \\
\text { téléphoniques } \\
\text { entre adhérents } \\
\text { envoi de fax sur } \\
\text { l'offre }\end{array}$ & $-\begin{array}{l}\text { développer une offre commune } \\
\text { pour BLO } \\
\text { mettre en place d'un système de } \\
\text { fax pour centraliser l'offre de } \\
\text { produits (de type Mercuriale) } \\
\text { mettre en place une tarification } \\
\text { unique avec Biocoop }\end{array}$ & $\begin{array}{l}\text { affirmation du Projet technico-économique } \\
\text { avec le développement des relations avec } \\
\text { Biocap, signature d'une convention } \\
\text { renforcement du Projet avec la sécurisation } \\
\text { des débouchés, l'augmentation des volumes } \\
\text { pour chaque producteur } \\
\text { engagement de nouveaux producteurs }\end{array}$ \\
\hline $\begin{array}{l}\text { réunions de } \\
\text { planification }(2 \\
\text { par an) } \\
\text { premier outil } \\
\text { d'offre en ligne }\end{array}$ & $-\begin{array}{l}\text { estimer la production de chacun } \\
\text { procéder à un réajustement de } \\
\text { ces prévisions. }\end{array}$ & $-\begin{array}{l}\text { Biocoop exprime sa demande de produit } \\
\text { auprès des producteurs qui répondent } \\
\text { favorablement ou pas selon la période } \\
\text { organisation et structuration de la filière à } \\
\text { partir de la planification de la production et du } \\
\text { partage d'information (via l'outil Mercuriale) } \\
\text { mieux faire valoir leur production biologique }\end{array}$ \\
\hline
\end{tabular}

Sur cette période, les producteurs, qui s'organisent pour planifier intelligemment leur production, s'engagent dans la mise en place d'un outil dit de «mercuriale de produits ». Cette première phase de structuration est décisive dans l'affirmation du Projet BLO et dans son évolution. Pendant près de trois ans, les producteurs communiquent par fax à partir de la liste commune de produits mise en place. L'objectif est que tout producteur participe à cette mercuriale de produits, et que le regroupement des offres des autres producteurs se fasse chez un producteur (pour un produit donné). Ce dernier est alors chargé de s'occuper de l'expédition auprès des magasins Biocoop. En même temps, les regroupements donnent une visibilité sur les productions des uns et des autres. Désormais, le système de gestion présente une première structuration et fonde des comportements que l'on peut considérer comme propres à BLO.

Nous avons choisi de faire un focus sur la mercuriale, dont le fonctionnement et l'évolution sont emblématiques des innovations mises en œuvre par l'association (Encadré 2).

\section{Encadré 2: L'outil mercuriale}

Le principe de fonctionnement de la Mercuriale est très basique, mais néanmoins novateur pour la profession agricole. Le principe est le suivant : chaque mois, un producteur centralisateur différent faxe à tous ses collègues une demande pour savoir quels sont leurs produits disponibles et les prix. Chaque producteur remplit le formulaire et le «refaxe» au centralisateur. Ce dernier reporte sur un même document toutes les offres, et envoie un fax à Biocap (plateforme et centrale d'achat de Biocoop) avec l'offre détaillée par producteur. Puis le centralisateur reçoit la commande de Biocap et envoie ce fax à tout le monde. En parallèle, BLO a travaillé sur la standardisation du calibrage et du colisage des produits. Sur cette base, Biocap passe commande à chaque producteur en lui adressant en même temps la commande complète, selon un principe de transparence totale. Biocap essaie de s'approvisionner un peu chez tout le monde pour ne pas créer des tensions entre producteurs. La coordinatrice de BLO intervient dans certains cas critiques (demande de Biocap inférieure à l'offre de BLO, mauvaise qualité d'une offre...).

Ces premiers arrangements en matière de production et de commercialisation s'avèrent à la fois structurants mais aussi révélateurs pour les acteurs de BLO de la capacité de leur collectif à pouvoir aller plus loin dans les fonctionnalités d'un dispositif d'intermédiation marchande. Ils prennent alors la mesure des possibilités de révision de leurs interactions entre eux, avec leurs clients habituels mais aussi avec de nouveaux clients, comme en témoignent ces propos : «Tout ce travail ensemble nous a rapprochés et on s'est dit qu'on pourrait aller plus loin. Donc on a créé une commission à ce moment-là. On a réfléchi à un système par internet. 
C'est là qu'est venu le projet de concevoir un site sur lequel on pourrait consulter nos produits et sur lequel chacun pourrait avoir des réponses. Chacun peut le gérer de chez lui pour sa part de production, tout en étant transparent avec les autres qui peuvent voir l'offre faite par les autres producteurs » (vice-président, 2012). Le développement des dispositifs de l'intermédiation commerciale allait accompagner ce que l'on peut considérer comme une phase de développement du projet collectif.

\subsection{Les dispositifs d'intermédiation marchande dans la phase de développement du projet collectif}

Avec le développement de l'activité et l'engagement de nouveaux agriculteurs dans le l'association, de nouveaux dispositifs simplement imaginés et entrevus sont mis en place. Le projet d'une offre en ligne est engagé et la mise en place d'un outil de facturation commune est associée à l'outil de planification.

Dans un souci d'efficience, la coordinatrice, en accord avec la direction, propose au CA de revoir les fonctionnalités et supports de l'outil «mercuriale » pour renforcer la structuration et donc la portée de l'action de BLO. Sur la base des premières réflexions engagées, elle suggère un nouveau dispositif fondé sur la conception d'un outil informatique et le recours à internet. Fin 2008, un administrateur, suggère que son fils, en BTS informatique, réalise son stage sur la réalisation de cet outil. Un groupe de travail est mis en place sur l'élaboration de l'outil. Cinq producteurs participent, sans que soient bien cernées à ce stade les fonctionnalités envisageables : «on veut alors un tableau à remplir avec des cases, comme avec le fax ...» (maraîcher, 2016). En avril 2009, l'outil d'offre en ligne finalisé est présenté en AG aux producteurs, à qui on explique le fonctionnement. Biocoop accueille cette évolution d'une offre en ligne avec enthousiasme. Parallèlement, BLO prend progressivement conscience de son pouvoir de marché. Le bureau décide de proposer ce dispositif à d'autres clients pour diversifier ses débouchés.

Le CA a défini des règles strictes de fonctionnement. « Il y a eu des discussions sur les règles, mais pas sur la légitimité de l'outil. Certains ( 3 ou 4 producteurs) sont en panique, car ils n'ont pas d'ordinateur et il a fallu les accompagner. Un seul réfractaire, ne s'y est jamais mis. Il passe encore aujourd'hui un coup de fil avec les acheteurs qui ne l'ont pas abandonné » (président, 2016). Mais l'acceptation de l'outil par les producteurs est favorisée par le fait « qu'ils se sentaient au bout du système de fax ». Tous sont favorables à l'outil au regard du projet politique de BLO, les rares réfractaires le sont par rapport à leur appréhension de l'informatique. Ce système ajoute aussi des contraintes. Par exemple, le producteur ne peut plus modifier son offre à partir de $9 \mathrm{~h} 30$, ni le client sa commande à partir de $17 \mathrm{~h} 30$. Mais à ce jour, la plateforme internet d'offre commune en ligne est opérationnelle et donne satisfaction: elle gère l'annonce des produits, la commande et la logistique d'expédition: " les volumes à destination des acheteurs sont planifiés par produit, par mois et par producteur. Il y a donc une réunion annuelle sur les légumes où les producteurs ont une vision d'ensemble des produits vendus par les collègues et une proposition de l'année suivante. Donc il y a vraiment une transparence et une connaissance de qui fait quoi dans l'organisation » (coordinatrice, mai 2015).

Parallèlement, BLO a développé un outil de facturation commune qui consiste, à partir du bon de livraison du producteur, à faire une facture client et une facture producteur. BLO travaille alors avec un informaticien pour une conception d'un outil ad hoc ${ }^{6}$. BLO a commencé

\footnotetext{
${ }^{6}$ Des questions institutionnelles, juridiques, fiscales émergent : peut-on le faire dans un cadre associatif ? A-t-on besoin de passer en coopérative ? En organisation de producteurs (OP) ? Les réponses des services juridiques et fiscaux leur confirment que BLO reste dans le cadre d'une gestion désintéressée. L'OP ou les coopératives coûtaient trop cher et obligeaient à faire des choses dont BLO n'avait pas besoin. Les adhérents voulaient rester sur une organisation simple, légère. Après avoir balayé l'ensemble des statuts possibles, BLO décide de rester en association. L'outil est opérationnel à partir de janvier 2007. Il permet de facturer et de payer les producteurs.
} 
à facturer les produits à partir de janvier 2007. «Pour beaucoup de producteurs, il fut très dur d'abandonner cette partie de leur métier » (coordinatrice, 2015). « Mais à partir de l'outil de facturation, le bureau de BLO envisage que cet outil commun puisse devenir un outil de gestion commerciale pour réaliser de la planification. Cet outil de gestion commerciale pouvait permettre non seulement de faire la facturation centralisée mais aussi la planification, et d'établir le lien entre les deux » (coordinatrice, 2015).

Ainsi, le projet associatif reposait sur un ensemble de dispositifs d'intermédiation commerciale qui allait accompagner son développement. La figure 1en fournit une synthèse visuelle.

Figure 1 : Les dispositifs de gestion de BLO en 2015

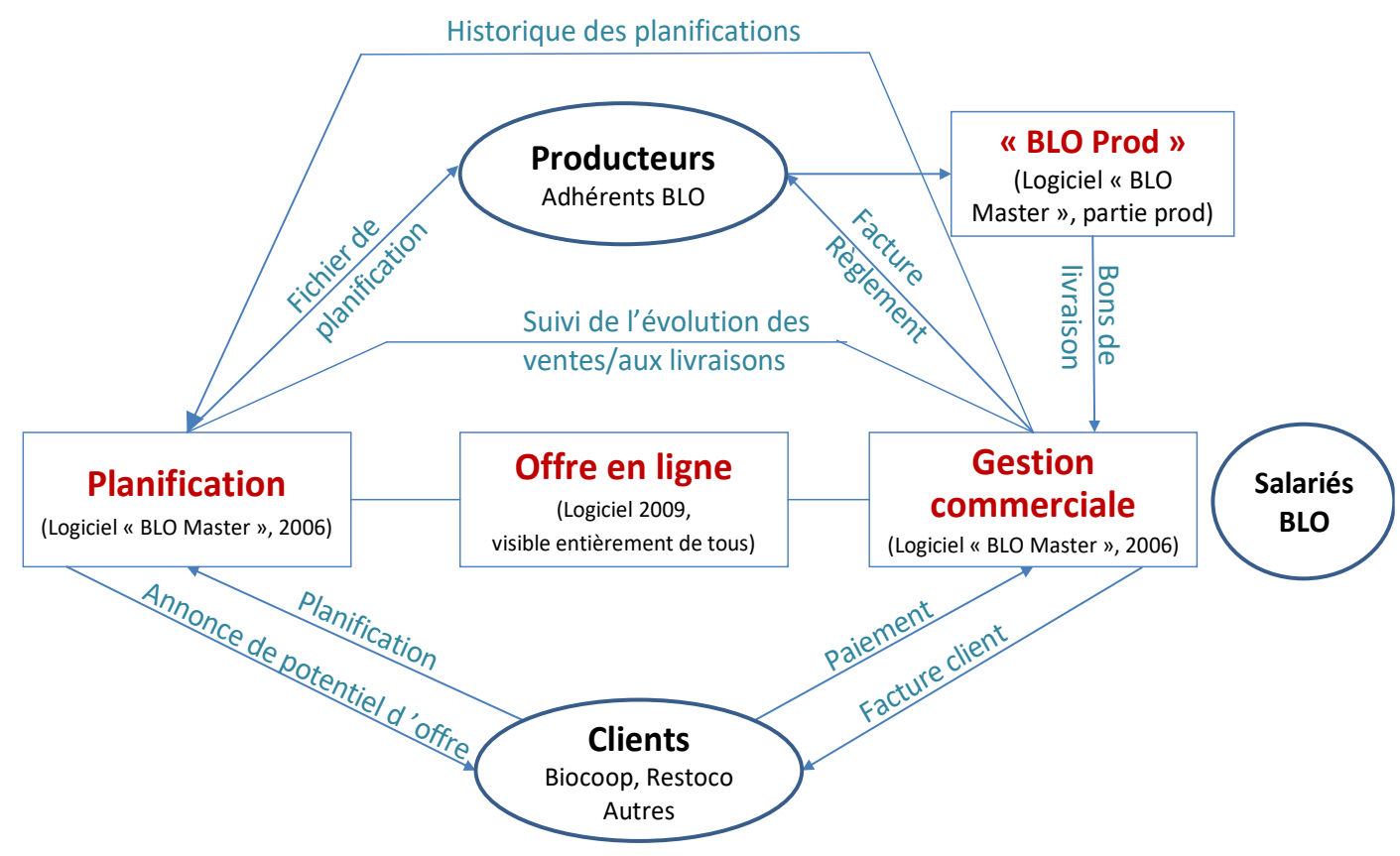

Offre en ligne : gère l'annonce des produits, la commande et la logistique d'expédition (date et lieu d'enlèvement). Cet outil a remplacé le fax en 2009).

Règlement des producteurs : tous les $10 \mathrm{j}$, Biocoop paie à $21 \mathrm{j}$.

" BLO Prod " : Logiciel de gestion commercial auquel a accès chaque producteur, pour ses propres productions

Tous ces instruments de gestion du projet collectif continuent d'évoluer: le logiciel de planification doit être reconstruit en 2018, le cahier des charges enregistre chaque année ou presque des modifications, etc. Des commissions constituées de producteurs volontaires, et en général d'une salariée, sont désignées en AG pour travailler sur ces évolutions.

\section{Analyse et enseignements}

Bien que l'approche par les dispositifs de gestion soit désormais bien inscrite dans le champ des sciences de gestion, elle demeure peu, voire pas, mobilisée en entrepreneuriat pour comprendre la phase d'émergence et de structuration d'un collectif d'acteurs autour d'un projet commun. Pourtant, comme le retiennent la TRS (Théorie de la Régulation Sociale) et la TEFP (Théorie de l'Entreprise Fondée sur le Projet) le Projet est constitutif du collectif et, à ce titre, les dispositifs du Projet le sont aussi. De cette recherche qui revêt un caractère exploratoire tentons de tirer des enseignements de portée générale et de les mettre en discussion. 


\subsection{L'interaction Projet-dispositif au cœur de l'action entrepreneuriale collective}

Devant l'indétermination profonde qui préside à tout processus entrepreneurial, la recherche en entrepreneuriat nous invite à explorer cet entrepreneur-ing (Hjorth, Holt, Steyaert, 2015). Nous croyons fécond, pour ce faire, de coupler les cadres théoriques existants sur les dispositifs de gestion à l'approche par le Projet, afin de donner de nouveaux éclairages sur les actions engagées pour se saisir d'une opportunité ou plus précisément pour l'exploiter en s'organisant collectivement. Pour dépasser l'analyse élémentaire de l'instrumentation de gestion habituelle en contexte entrepreneurial (avec notamment le modèle d'affaires, le business plan...), nous avons privilégié une approche fondée sur le couple projet entrepreneurial / dispositif. Pour cela, il était logique d'envisager leurs interactions au fil de la vie du Projet. La posture clinique s'imposait dans le cadre d'une recherche-accompagnement menée sur plusieurs années. En effet, s'intéresser à l'émergence et au développement d'un collectif, c'est s'intéresser à un projet dans sa singularité, conformément à la perspective ontogénétique (et à ce titre développementale, subjective et multidimensionnelle) de la TEFP (Desreumaux et Bréchet, 2009).

Avec l'histoire de BLO, nous voyons combien l'action est à entreprendre et à construire. Si l'on retient qu'une opportunité entrepreneuriale était identifiable en lien avec le développement des marchés bio, nous ne pouvons pas en rester à ce constat. Il faut s'intéresser à la façon dont les acteurs s'en saisissent. C'est parce que des acteurs font preuve d'initiative et s'engagent dans un projet d'action commune qu'elle existe ; elle se précise dans des produits et de services. Il s'agit donc de s'intéresser à l'action collective qui s'invente et se construit, aux processus d'organisation (à l'« organizing » pour reprendre les termes de Gartner, 1985 : 697) qui s'instruisent dans le cadre du projet collectif, observable avec les dispositifs qui en accompagnent la conception et le déploiement.

Répondre à la question de savoir comment les dispositifs gestionnaires rendent possible le projet collectif, suppose de repérer les interactions et impacts des dispositifs du Projet avec l'action entrepreneuriale. La distinction entre le projet politique, le projet économique et le projet organisationnel permet une lecture analytique de ce qui se joue. Le tableau 3 organise une synthèse de ce que nos développements précédents ont abordés.

Tableau 3 : Les interactions et impacts des dispositifs du Projet avec l'action entrepreneuriale

\begin{tabular}{|c|c|c|}
\hline $\begin{array}{l}\text { Les dimensions du } \\
\text { Projet }\end{array}$ & Les dispositifs du Projet & $\begin{array}{l}\text { Interactions et impacts des dispositifs avec l'action } \\
\text { entrepreneuriale }\end{array}$ \\
\hline $\begin{array}{l}\text { Projet politique } \\
\text { - aspects éthiques et } \\
\text { politiques - }\end{array}$ & $\begin{array}{l}\text { - } \quad \text { Charte comme expression des valeurs } \\
\text { et des principes } \\
\text { - } \quad \text { Cahier des charges comme } \\
\text { opérationnalisation de la charte } \\
\text { - } \quad \text { Bureau, CA, comité technique }\end{array}$ & $\begin{array}{l}\text { - La charte : le référent dans l'action politique, } \\
\text { technique, productive et commerciale } \\
\text { - } \quad \text { Le cahier des charges : les règles à respecter } \\
\text { pour les cultures et le management } \\
\text { - La vie de la gouvernance }\end{array}$ \\
\hline $\begin{array}{l}\text { Projet économique } \\
\text { - aspects techniques } \\
\text { et économiques - }\end{array}$ & $\begin{array}{ll}\text { - } & \text { Mission(s) et métier (s) respectueux } \\
\text { des axes du Projet politique (sur la } \\
\text { commercialisation, l'éthique...) } \\
\text { - } \quad \text { Mercuriales de prix et de produits } \\
\text { - } \quad \text { Outil de planification / facturation } \\
\text { - } \quad \text { Offre en ligne }\end{array}$ & $\begin{array}{l}\text { - La vigilance du bureau sur l'équilibre entre } \\
\text { BLO et les activités de chaque producteur } \\
\text { - } \quad \text { Les outils d'intermédiation marchande } \\
\text { constitutifs du cœur du Projet productif BLO }\end{array}$ \\
\hline $\begin{array}{l}\text { Projet organisationnel } \\
\text { - aspects } \\
\text { d'organisation et } \\
\text { d'animation - }\end{array}$ & $\begin{array}{l}\text { - Travail de coordination assuré par une } \\
\text { personne et des groupes de travail } \\
\text { thématiques (label, semences, RHD...) } \\
\text { - Plateformes d'échanges et de } \\
\text { coopérations entre producteurs et des } \\
\text { regroupements de produits }\end{array}$ & $\begin{array}{l}\text { - Un ajustement des outils et des pratiques } \\
\text { d'échanges d'information et de concertation } \\
\text { assuré par la chargée de Projet coordinatrice } \\
\text { - } \quad \text { Une évolution des relations entre producteurs } \\
\text { - } \quad \text { Une évolution des relations avec les clients } \\
\text { - } \quad \text { Plus de confiance et de réciprocité dans les } \\
\text { échanges }\end{array}$ \\
\hline
\end{tabular}

La distinction que nous avons précédemment opérée entre la phase d'émergence et la phase de développement du projet collectif témoigne, nous semble-t-il, de la nécessité d'intégrer la question de la temporalité. L'analyse longitudinale a permis de mettre en évidence les effets de leviers des dispositifs sur la formation du projet entrepreneurial, sur le délicat passage du 
projet d'entreprendre au projet d'entreprise (Bréchet, 1994) si l'on veut bien accepter cette distinction. Sans ces dispositifs, le projet entrepreneurial de BLO aurait eu du mal à se concrétiser. En même temps, la révision inventive des dispositifs pour accompagner leurs pratiques a favorisé l'évolution du Projet et des pratiques.

La temporalité doit donc être intégrée à l'analyse. L'évolution des dispositifs est graduelle, permettant l'instauration d'un climat de confiance et de coopération, favorisant par exemple l'implémentation de la mercuriale puis d'un outil d'offre en ligne. Une telle démarche a nécessité discussions, débats, négociations entre acteurs; elle a aussi supposé des phases d'expérimentation. Aujourd'hui, le dispositif de gestion fondé sur l'outil en ligne promeut une grande transparence et la tenue des engagements. Il est le vecteur d'une dynamique de confiance mutuelle. Les producteurs adhérents à BLO l'utilisent quotidiennement pour connaître l'état de l'offre de légumes, pour mieux définir leur prix et répondre aux demandes des clients (réseau Biocoop, grossistes, Marché d'intérêt régional et national, GMS...). Cet effort de régulation collective est particulièrement intéressant à souligner pour des organisations agricoles qui ne sont pas particulièrement familières de telles pratiques collectives.

Dans le projet entrepreneurial collectif ouvert qu'était celui des premiers acteurs de BLO, les dispositifs gestionnaires ont permis aux producteurs impliqués de produire des connaissances sur eux-mêmes, sur leurs exploitations, sur leurs pratiques et surtout de les partager. Les dispositifs ont favorisé la transparence et renforcé ainsi la confiance entre les producteurs, de même qu'entre les producteurs et les clients. Les dispositifs de l'intermédiation commerciale ont ainsi conduit les producteurs à envisager de nouvelles modalités d'organisation pour vendre directement aux clients locaux et de manière collective. C'est dans l'interaction projet-dispositif que s'est renforcée, et peut-être qu'est née, la prise de conscience de leur capacité à s'autonomiser des régulations dominantes des marchés agricoles. Les dispositifs ont accompagné le développement de leur action collective et favorisé des apprentissages du vivre et travailler ensemble, dont il faut reconnaître qu'ils sont au fondement de leur capacité d'action collective. Les dynamiques de construction des relations et des connaissances permises par les dispositifs du Projet les ont aussi conduits à préciser leurs orientations de développement et parfois les remettre en cause. La charte et le cahier des charges portent la trace de ces évolutions au fil de la vie du Projet.

Enfin, rappelons que, loin de s'en remettre à un fournisseur d'outils, les porteurs du projet collectif BLO se sont dotés d'un outil sur mesure dans ses principes, dans son support technique comme dans ses pratiques. La structuration du Projet BLO s'est donc faite sur la base de nouvelles voies de coopération agricole, en dehors du cadre coopératif dominant, en même temps qu'elle était supportée par des dispositifs spécifiques et fondés sur des interactions nouvelles entre acteurs et outils.

\subsection{Le travail de régulation : du projet d'entreprendre au projet d'entreprise}

Notre posture méthodologique à caractère clinique nous a permis d'étudier précisément et dans la durée, le travail de régulation engagé pour faire vivre le projet entrepreneurial collectif. Affirmer que BLO a su tirer parti de l'opportunité entrepreneuriale du marché du Bio, et plus particulièrement de la demande de Biocoop, est court en termes d'arguments; encore faut-il analyser comment l'association a agi, comment elle a su construire une capacité d'action collective. L'action collective engage un travail de régulation et le contexte entrepreneurial, donc de l'émergence et de la construction d'un collectif, est de ce point de vue instructif.

La genèse du projet BLO révèle la diversité des actions menées par les acteurs pour structurer leur projet collectif initial. Sans l'engagement dans des phases d'expérimentation où les acteurs ajustent leurs outils et pratiques, où ils rencontrent des résistances qui les font douter de leur capacité à se doter de règles propres, le projet BLO ne se serait peut-être pas affirmé. Or précisément, c'est par l'instrumentation déployée que nous pouvons appréhender comment les acteurs construisent leur action, en même temps qu'ils se découvrent et s'interrogent sur leur 
Projet initial. Avec les actions rendues possibles par l'instrumentation mise en place, les acteurs constatent les écarts entre le Projet-en-idée et le Projet-en-acte.

Les phases d'émergence, mais aussi de développement, se montrent par ailleurs riches en apprentissages. Elles amènent les acteurs à reconsidérer les régulations et les règles régissant leurs pratiques opérationnelles - à la fois dans leur mise en production et dans leur commercialisation-. Ils peuvent alors repenser leur Projet, c'est-à-dire réviser les principes et les critères que l'on va retrouver dans la charte et le cahier des charges. Dans la phase d'émergence, le recours à des outils simples pour coordonner l'offre commune, dans un contexte de ressources et de moyens limités, a permis à BLO de prendre progressivement conscience de la singularité de ses pratiques et de son potentiel pouvoir de marché. Mais ce pouvoir supposait un travail de régulation (Tableau 4), récurrent, stimulé par les producteurs les plus décidés, les plus opiniâtres. Le tableau 4 reprend synthétiquement les principales étapes du travail de régulation, et ce qu'il recouvre.

Tableau 4 : Les principales étapes repérées dans le travail de régulation de 1997 à 2010

\begin{tabular}{|c|c|c|}
\hline Acteurs concernés & Objectifs & Travail de régulation \\
\hline $\begin{array}{l}\text { 1997- } 2000 \\
5 \text { / } 6 \text { agriculteurs } \\
\text { adhérents de BLO } \\
\text { Demande de } \\
\text { BIOCOOP }\end{array}$ & $\begin{array}{l}\text { - Organiser la facturation des } \\
\text { produits pour passer de } 50 \\
\text { factures à une facture }\end{array}$ & $\begin{array}{ll}- & \text { Réunions de travail pour discuter des } \\
\text { orientations du Projet ; } \\
\text { - }\end{array}$ \\
\hline $\begin{array}{l}\text { 2001-2002 } \\
\text { Une dizaine } \\
\text { d'adhérents pour BLO } \\
\text { Demande de BIOCAP }\end{array}$ & $\begin{array}{l}\text { - Organiser la production via } \\
\text { l'échange de fax, } \\
\text { producteurs et BLO } \\
\text { Mettre en place une } \\
\text { planification avec BIOCAP }\end{array}$ & $\begin{array}{ll}\text { - } & \text { Travail de recensement des fermes, des } \\
\text { productions et techniques (phase } \\
\text { d'apprentissage mutuel) } \\
\text { - } \\
\text { Réalisation d'un livret de promotion des } \\
\text { adhérents de BLO et de leurs produits } \\
\text { - } \quad \begin{array}{l}\text { Harmonisation de la qualité des produits et de } \\
\text { leurs prix }\end{array} \\
\text { - } \begin{array}{l}\text { Début de la mercuriale de produits (pour fixer } \\
\text { les volumes et tarifs de commercialisation) }\end{array}\end{array}$ \\
\hline $\begin{array}{l}2002-2004 \\
\text { Une vingtaine } \\
\text { d'adhérents pour } \\
\text { BLO }\end{array}$ & $\begin{array}{l}\text { - Mettre en place des outils } \\
\text { pour la commercialisation, } \\
\text { l'expédition et début de } \\
\text { palettisation collective } \\
\text { Favoriser la réactivité des } \\
\text { producteurs / demande des } \\
\text { acheteurs }\end{array}$ & 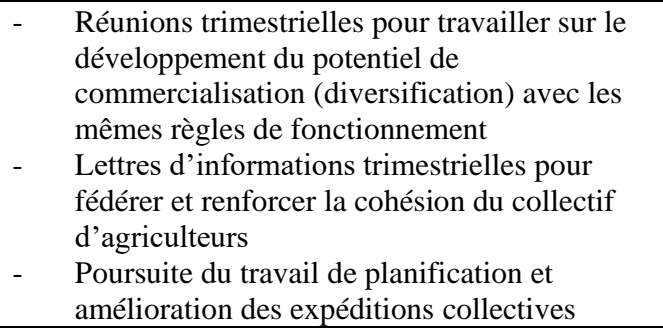 \\
\hline $\begin{array}{l}2004 \text { à } 2006 \\
\text { De nouveaux } \\
\text { adhérents pour } \\
\text { BLO } \\
\text { Soutien de la Draaf }\end{array}$ & $\begin{array}{ll}\text { - } & \begin{array}{l}\text { Développer les points de } \\
\text { regroupement de l'offre; } \\
\text { renforcer l'harmonisation } \\
\text { des prix (échanges via } \\
\text { téléphone et Fax) }\end{array} \\
\text { Mettre en place une } \\
\text { facturation commune } \\
\text { - } & \text { Lancer le logo BLO } \\
\text { - } & \text { Elaborer un cahier des } \\
\text { charges de production }\end{array}$ & $\begin{array}{l}\text { - Elaboration du programme d'action autour } \\
\text { d'une stratégie plus ambitieuse de structuration } \\
\text { de la filière Fruits et Légumes (avec soutien de } \\
\text { la Draaf) } \\
\text { - Déploiement sur une plus grande échelle } \\
\text { territoriale des principes de coopération et de } \\
\text { régulation mis en place entre les adhérents }\end{array}$ \\
\hline $\begin{array}{l}2006-2007 \\
\text { De nouveaux } \\
\text { adhérents pour BLO }\end{array}$ & 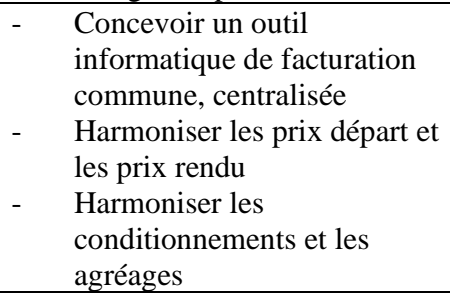 & $\begin{array}{l}\text { - } \quad \text { Affirmation du Projet éthico-politique vis-à- } \\
\text { vis de l'environnement ; } \\
\text { - } \quad \text { Besoin de définir de nouvelles règles } \\
\text { d'organisation avec l'arrivée d'adhérents } \\
\text { - } \quad \text { Conception d'un outil informatique spécifique } \\
\text { (pour à partir du bon de livraison du } \\
\text { producteur, élaborer une facture client et une } \\
\text { facture producteur) }\end{array}$ \\
\hline $2008-2010$ & - $\quad$ Développer les & - $\quad$ Conception d'un nouveau dispositif en ligne \\
\hline
\end{tabular}




\begin{tabular}{|l|l|l|}
\hline $\begin{array}{l}\text { Une cinquantaine } \\
\text { d'adhérents }\end{array}$ & $\begin{array}{l}\text { fonctionnalités d'un logiciel } \\
\text { vers un fonctionnement } \\
\text { d'une offre en ligne }\end{array}$ & $\begin{array}{l}\text { pour soutenir la commercialisation vers de } \\
\text { nouveaux débouchés ; } \\
\text { Sollicitation d'un informaticien pour élaborer } \\
\text { le logiciel d'offre en ligne }\end{array}$ \\
\hline
\end{tabular}

Revenons une dernière fois sur la singularité des régulations mises en œuvre. Bien que les travaux sur les dispositifs de gestion soulignent combien ces outils peuvent être sources d'isomorphisme mimétique entre organisations de nature, métiers, secteurs différents (Chiapello et Gibert, 2013; Grimand, 2016) il est en effet intéressant de constater que chez BLO, les dispositifs se fondent sur des régulations originales que les producteurs de l'association veulent faire prévaloir. Par les usages nouveaux qu'ils permettent, ces dispositifs suscitent des effets inattendus et «façonnent» le projet entrepreneurial collectif, mais aussi participent de la capacité de l'organisation à exploiter l'opportunité entrepreneuriale initiale tout en respectant les valeurs que les producteurs veulent défendre.

L'expérience de BLO nous permet, enfin, d'approfondir cette question constitutive des débats au sein du paradigme de l'opportunité (Chabaud et Messeghem, 2010). Ireland, Hitt, Sirmon (2003 : 966) soulignent cette tension majeure entre la capacité à identifier des opportunités et la capacité à les exploiter. Dans un contexte entrepreneurial, cette capacité est précisément à construire, elle requiert un travail de régulation entre acteurs pour s'accorder à la fois en pensée et en actes. Ce travail a permis la conception d'un dispositif innovant de régulation marchande, autour de la gestion du couple offre-demande (avec les aspects de planification et les divers interfaçages entre acteurs), mais aussi des choix organisationnels et managériaux qui font vivre le collectif sans l'assujettir aux régulations marchandes dominantes qui le dénatureraient. Les dimensions économiques et politiques du Projet sont liées. Et c'est dans et par l'action qu'elles se manifestent.

\subsection{La perspective d'une théorie de l'action fondée sur le projet}

Aux termes de cette contribution, nous souhaitons finir sur les apports de la recherche en ce qui concerne la théorisation de l'action. Nous les avions annoncés d'ordre théorique, à travers le rapprochement de deux champs de recherche : celui de l'instrumentation gestionnaire et celui de la théorie de l'entreprise fondée sur le Projet (TEFP), dans le cadre de la Théorie de la régulation sociale (TRS). Les rapprochements entre la TEFP et la TRS ont déjà opéré sur la base de l'idée-force que les collectifs naissent des projets qu'ils nourrissent'.

La TRS fait reposer l'existence des règles sur l'action collective. J.-D. Reynaud le dit souvent, les règles n'ont de sens que rapportées aux fins d'une action commune, c'est-à-dire d'un projet commun; se joindre à une action collective, c'est accepter d'entrer dans le jeu qui maintient et transforme les règles, c'est accepter de vivre une régulation. Cette importance du Projet pour comprendre l'action collective est encore peu intégrée, que ce soit dans les disciplines de l'économie, de la sociologie ou du management. Le lien entre le Projet et les dispositifs du Projet permet d'éclairer cette importance.

Une première idée d'évidence peut être évoquée, celle qui pose que le Projet demande à être imaginé, conçu et déployé, ce qui signifie des démarches d'élaboration de son contenu et d'accompagnement de son déploiement, ces deux aspects étant liés. On pourrait associer cette idée à celle habituellement mise sous l'étiquette des démarches de projet. Ainsi, l'élaboration et l'application de la charte et du cahier des charges chez BLO impliquent des instances et pratiques d'élaboration et de suivi de leur respect, supportés par des dispositifs. La seconde idée, que nous privilégions, est celle qui établit que le Projet, en tant qu'ensemble de règles d'ordre politique et stratégique, fonde le collectif. Cette compréhension du Projet est directement à rapprocher de ce nous dit J.-D. Reynaud à propos de la règle : « Ce qui fait qu'une règle est respectée, c'est avant tout qu'elle permet une action collective. Cela signifie deux choses : d'une part qu'elle est acceptée par les autres comme pouvant diriger cette action

\footnotetext{
${ }^{7} \mathrm{Cf}$. pour une synthèse Bréchet et Desreumaux, 2012.
} 
collective ; d'autre part qu'elle permet d'atteindre l'objet de l'action collective » (Reynaud, $2005)^{8}$.

La charte et le cahier des charges sont considérés dans cette contribution comme les règles qui expriment le Projet. Ils représentent, à ce titre, les dispositifs d'instrumentation du Projet : ils en révèlent le contenu et en permettent l'actualisation. Mais ce que nous voulions mettre en évidence, c'est que l'application de la charte et du cahier des charges s'accompagne aussi de dispositifs de gestion de l'intermédiation marchande (planification, vente en ligne, marque...) qui structurent la vie du collectif et rendent possible sa volonté de faire exister son Projet dans le marché. Le Projet BLO vit par ce que certains nomment les agencements marchands (Cochoy, Trompette et Araujo, 2016; LeVelly et Dufeu, 2016) qu'il prévoit et fait vivre. La charte et le cahier des charges chez BLO forment un système intégré de principes et de critères, dont la cohérence même fonde l'originalité du Projet BLO dans le monde de l'agriculture biologique (Dufeu, Le Velly, Bréchet et Loconto, 2020). Mais ce Projet à vocation commerciale ne peut exister ni se comprendre sans l'intermédiation marchande qu'il organise. Les règles que pose le Projet par la charte et le cahier des charges ne pourraient vivre sans les dispositifs marchands, les outils élaborés et animés dans et par les pratiques collectives qui rendent effectivement possible le respect des règles posées comme référent et comme cadrage de l'action. Quand on parle règle, il faut toujours avoir à l'esprit les dispositifs homme-machine qui en permettent le jeu, et qui sont aussi porteurs d'effets propres sur les comportements en même temps que dotés d'inertie du fait des aménagements et des coûts qu'ils engagent.

Si l'on retient que toutes les règles cadrent et encadrent l'action, structurent des jeux organisationnels, il faut par ailleurs reconnaître que certains jeux sont d'ordre politique et stratégique (ou du Projet) et d'autres d'ordre plus opérationnel. Ces deux ordres ne sont nullement indépendants mais interdépendants, dans des relations de dépendance croisée entre fins et moyens de l'action. Cette idée trouve une autre expression dans les perspectives duales couramment retenues dans le domaine du management: opposition entre logique de développement et logique d'exploitation dans la vie de l'entreprise ; opposition entre logique (ou mode) stratégique et logique opérationnelle, ce qui revient à distinguer la gestion stratégique de la gestion courante ; opposition entre création de potentiel et exploitation du potentiel de l'entreprise, opposition à directement rapprocher de la précédente, le mode stratégique s'associant à la création de potentiel et le mode opérationnel à son exploitation.

Le lien établi entre le Projet, les règles et les dispositifs n'est pas sans mobiliser les problématiques habituelles par lesquelles on aborde l'instrumentation gestionnaire, mais ici il s'agit bien de mettre en évidence l'aspect politico-stratégique des dispositifs qui permettent d'affirmer et de faire vivre le projet entrepreneurial collectif. Bizarrement, la littérature sur l'instrumentation de gestion s'est moins intéressée à cet aspect qui engage l'ordre des fins, comme si la gestion, à l'instar de l'économie, était plus intéressée à l'ordre des moyens. Et pourtant, la littérature académique, autant que professionnelle, multiplie les grilles diverses d'analyse stratégique, avec, de nos jours, l'omniprésence du modèle d'affaires (Germain et Jacquemin, 2017). Mais le lien n'est pas fait avec la constitution des collectifs. C'est cette médiation vers l'action collective qu'ajoute le Projet en tant qu'il représente une perspective d'action commune qui va se concrétiser par une communauté de règles vécues, donc en tant qu'il représente des règles que le collectif imagine et conçoit, qu'il va faire vivre et par lesquelles il existe. Le Projet constitue le collectif en tant qu'il recouvre des règles et des régulations qui sont au fondement d'une capacité d'action commune. Ces compréhensions sont au cœur de la TRS, autant que de la TEFP.

\footnotetext{
${ }^{8}$ Cette citation est extraite d'un entretien avec J.-D. Reynaud qui était invité à préciser une assertion qu'il a fait sienne selon laquelle « il n'y a pas de règle sans projet » (cf. Reynaud, 2005).
} 


\section{Conclusion}

A l'issue de nos travaux, force est de constater la pertinence de l'approche par les dispositifs de gestion à la fois pour s'intéresser à la constitution du collectif et pour analyser comment des individus conçoivent de manière inventive l'action collective. A travers l'étude des liens projet collectif-dispositif de gestion, on comprend que le projet collectif entrepreneurial trouve dans les dispositifs de gestion le support indispensable à l'action collective qu'il promeut et qui le manifeste. L'action collective fondée sur un Projet ne va pas sans le travail de régulation instrumenté qui la rend possible.

Cette recherche s'inscrivait par nature dans une posture clinique, qui est au fondement de l'approche pragmatiste (Dewey, 2011, 2006), comme de la TRS, posture que revendique la TEFP'. Quand on considère l'action en tant que régulation, il s'agit toujours de l'action et de la régulation dans leur singularité. Une singularité qui s'exprime dans un rapport unique aux personnes, à l'espace et au temps (Boutinet et Bréchet, 2014). L'expérience BLO, sur laquelle nous nous sommes appuyés, témoigne de ces ancrages qui permettent de comprendre l'émergence et l'existence d'une action collective originale et innovante.

Dans le cas présent, nous avons mené une investigation des pratiques collectives ${ }^{10}$ par lesquelles un collectif d'individus s'est doté d'un projet singulier d'exister commercialement dans le marché. En choisissant d'explorer les liens Projet collectif - dispositif de gestion dans l'analyse de la genèse du projet entrepreneurial collectif de BLO, cette recherche revêtait un caractère exploratoire. De notre point de vue, elle engage une réflexion novatrice. Dans le cadre de l'approche par le Projet, au sens de projet collectif, il nous faut souligner une difficulté. Elle tient au fait que le concept de Projet, posé au fondement des collectifs, vise à saisir l'action dans sa nature profondément multidimensionnelle et l'on pourrait dire dans sa complexité. Cela rend difficile, et délicate à manier, la posture analytique dont nous avons exploré la mobilisation en distinguant les dimensions politiques, économiques et organisationnelles. On mesure que des voies d'approfondissement de la réflexion seront nécessaires pour affiner l'effort de théorisation.

\section{Bibliographie}

AGGERI F. et LABATUT J. (2010), «La gestion au prisme de ses instruments. Une analyse généalogique des approches fondées sur les instruments de gestion », Finance Contrôle Stratégie, vol.13, ${ }^{\circ} 3$, septembre, p. 5-37

ASQUIN A., CONDOR R. et SCHMITT C. (2011), "Pour la mobilisation de la notion de Projet dans la recherche en entrepreneuriat », Revue de l'entrepreneuriat, vol. 10, n॰2, p.7-14.

BERRY M. (1983), L'impact des instruments de gestion sur l'évolution des systèmes humains, Texte de synthèse au rapport collectif pour la DGRST fait par le CRG, 49p.

BOUTINET J.-P. (1990 / 2012), Anthropologie du Projet, PUF.

BOUTINET J.-P. et RAVELEAU B. (2011), "Questionnement autour du Projet entrepreneurial », Revue de l'entrepreneuriat, vol.10, n`2, p.15-28

BOUTINET J.-P. et BRECHET J.-P. (2014), Logiques de projet, logiques de profit. Convergences ou oppositions, Lyon, Chronique Sociale.

BRECHET J.-P. (1994), Du Projet d'entreprendre au Projet d'entreprise», Revue Française de Gestion. n' 99 , p. 5-15.

BRECHET J.-P. (2008), « Le regard de la théorie de la régulation sociale de Jean-Daniel Reynaud », Revue française de gestion, vol. 34, n 184 , p. 13- 25.

\footnotetext{
${ }^{9} \mathrm{Cf}$. aussi sur ce point Marchesnay (2008).

${ }^{10}$ Dans cet article, nous avons dû délaisser des aspects essentiels qui touchent aux recrutements, aux rémunérations, à la prise de décision, la délégation ou bien encore à la division du travail au sein de la structure d'animation. Ces aspects seront développés dans une autre contribution.
} 
BRECHET J.-P. et DESREUMAUX A. (2012), Le projet dans l'action collective, Encyclopédie des Ressources Humaines, Paris, Vuibert, p. 1130-1136

BRECHET J.P., EMIN S. et SCHIEB-BIENFAIT N. (2014), « La recherche-accompagnement : une pratique légitime ? », Revue Finance Contrôle Stratégie, vol.17, n², mis en ligne le 19 juillet 2014, consulté le 30 mai 2018. URL : http://journals.openedition.org/fcs/1477 ; DOI : 10.4000/fcs. 1477 .

BRECHET J.-P. et SCHIEB-BIENFAIT N. (2006), Projets et pouvoirs dans les régulations concurrentielles : la question de la morphogenèse d'une filière d'agriculture biologique, Revue d'Economie Industrielle, ${ }^{\circ} 113$, p. 1-20.

BRECHET J-P. et SCHIEB-BIENFAIT N. (2011), «L'entrepreneuriat confronté au pluralisme théorique : la nécessité d'une Project-Based View, Revue de l'entrepreneuriat, vol.10, $\mathrm{n}^{\circ} 2$, p.29-44

BRECHET J-P., SCHIEB-BIENFAIT N. et DESREUMAUX A (2009), "les figures de l'entrepreneur dans une théorie de l'action fondée sur le projet", Revue de l'entrepreneuriat, vol. $8, \mathrm{n}^{\circ} 1$, p. $37-54$.

CHABAUD D., MESSEGHEM K. (2010), « Le paradigme de l'opportunité : des fondements à la refondation », Revue française de Gestion, vol.7, n²06, p. 93-112.

CHIAPELLO E. ET GIBERT P. (2013), Sociologie des outils de gestion, Paris, La Découverte.

COCHOY F., TROMPETTE P. et ARAUJO L. (2016), « From market agencements to market agencing: an introduction », Consumption, Markets \& Culture, vol. 19, n¹, 3-16.

COOK M.L. et PLUNKETT B. (2006), "Collective Entrepreneurship : an Emerging Phenomenon in Producer-Owner Organizations », Journal of Agricultural \& Applied Economics vol. 38, n², February.

DAVID A. (1998). "Outils de gestion et dynamique du changement », Revue Française de Gestion, septembre-octobre, ${ }^{\circ} 120$, p. 44-59.

DAVID A., HATCHUEL A et LAUFER R. (2000), Les nouvelles fondations des sciences de gestion, Paris, Vuibert.

DE VAUJANY F.X., (coord.) (2005), De la conception à l'usage : éléments d'un management de l'appropriation des objets et outils de gestion, Editions EMS, Collection «Questions de Société ».

DE VAUJANY F.X. (2006), "Les outils de gestion; vers de nouvelles perspectives théoriques », Management \& Avenir, vol.3, n 9 , p.107-108.

DESREUMAUX A. et BRECHET J.-P. (2009), «Quels fondements pour les théories de la firme ? Plaidoyer pour une théorie artificialiste de l'action collective fondée sur le Projet », in BAUDRY B. et DUBRION B., Analyses et transformations de la firme. Une approche pluridisciplinaire, Paris, La Découverte, p. 61-89.

DEWEY J. (2011), La formation des valeurs, Paris La Découverte

DEWEY J. (2006), Logique, la théorie de l'enquête, Paris, PUF.

DUFEU I., DUCHAINE S., MC NAMARA T., MORVAN C. (2016), "L'agriculture biologique contribue-t-elle au bien-être? », Éthique et Economique/Ethics and Economics, vol.13, ${ }^{\circ} 1$, p. 39-57.

DUFEU I., LE VELLY R., BRÉCHET J.P., LOCONTO A., (2020), Can standards save organic farming from conventionalisation? Dynamics of collective projects and rules in a French organic producers' organization, Sociologia Ruralis, 60 (3), pp 621-638

EMIN S., SCHIEB-BIENFAIT N. (2013), « De la pertinence des approches par le projet pour analyser les processus entrepreneuriaux collectifs : une étude de cas sur le territoire de la Confédération Paysanne", Revue de l'entrepreneuriat, vol. 12, n¹-2, p.15 à 42GARTNER W., (1985), «A conceptual Framework for describing the phenomenon of new venture creation », Academy of Management Review, vol.10, nº 4, p.696-706.

GARTNER W. (1995), "Aspects of organizational Emergence», in I. Bull, H. Thomas, G. Willard (ed.), Entrepreneurship : perspectives on theory building, New York, Pergamon 
GERMAIN O., JACQUEMIN A. (2017), «Voies et voix d'approches critiques en entrepreneuriat », Revue de l'Entrepreneuriat, vol. 16, n 1, p.7-18.

GRIMAND A. (2016), «La prolifération des outils de gestion : quel espace pour les acteurs entre contrainte et habilitation ? », Recherche en Sciences de Gestion, $\mathrm{n}^{\circ} 112$, p.173-196.

HATCHUEL A. (2000), "Quel horizon pour les sciences de gestion ? Vers une théorie de l'action », in DAVID A., HATCHUEL A. et LAUFER R.., Les nouvelles fondations des sciences de gestion, Paris, Vuibert.

HATCHUEL A., MOISDON J.-C. (1993), «Modèles et apprentissage organisationnel », Cahiers d'Economie et Sociologie Rurales, $\mathrm{n}^{\circ} 28, \mathrm{p} .17-32$.

HATCHUEL A., WEIL B. (1992), L'expert et le système, Editions Economica.

HJORTH D., HOLT R., STEYAERT C. (2015), « Entrepreneurship and process studies », International Small Business Journal, vol.33, nº, p. 599-611.

IRELAND R.D. HITT M.A., SIRMON D.G. (2003), « A model of Strategic Entrepreneurship : The Construct and its Dimensions », Journal of Management, vol.29, n6, p.963-989.

LE VELLY R., DUFEU I. (2016), " Alternative food networks as market agencements: exploring their multiple hybridities », Journal of Rural Studies, $\mathrm{n}^{\circ} 43$, p. 173-182.

LE VELLY R., DUFEU I. et LE GREL L. (2016), " Les systèmes alimentaires alternatifs peuvent-ils se développer commercialement sans perdre leur âme ? Analyse de trois agencements marchands », Economie Rurale, Novembre-décembre, vol. 356, p. 31-47.

MARCHESNAY M. (2008), "Le cas entrepreneurial : retour à la maïeutique », Revue Française de Gestion, vol. 5, n 185 , p.175-189.

MC ELWEE G. (2006), « Farmers as entrepreneurs: Developing competitive skills », Journal of developmental Entrepreneurship, vol. 11, n³, p.187-206.

MC ELWEE G. (2008), «A taxonomy of entrepreneurial farmers », International Journal of Entrepreneurship and Small Business, vol. 6, $\mathrm{n}^{\circ} 3$, J June, p.465-478.

MOISDON J.-C. (dir.) (1997), Du mode d'existence des outils de gestion, Paris, Seli Arslan.

MOISDON J.-C. (2005), «Comment apprend-on par les outils de gestion? Retour sur une doctrine d'usage », in TEULIER R. et LORINO P. (eds), Entre connaissance et organisation: l'activité collective, La Découverte, p.239-259.

NGIJOL J. (2015), «Isräel M. Kirzner: les opportunités au cœur de la dynamique entrepreneuriale », Revue de l'Entrepreneuriat, ${ }^{\circ} 4$, vol.14, p.74-99.

REYNAUD J.-D. (1989 / 1997), Les règles du jeu ; l'action collective et la régulation sociale, 3ème Edition, Paris, Armand Colin.

REYNAUD J.-D. (2005), « Dans une relation de régulation il y a une relation de pouvoir », entretien, in Les organisations, état des savoirs, Editions Sciences humaines, p. 155-162.

REYNAUD J.-D. (2008), « Qu'est-ce qu'une communauté de projet? » in Vrancken D., Dubois C. et Schoenaers F., Penser la négociation. Mélanges en l'honneur de Olgierd Kuty, De Boeck Supérieur, p. 189-192.

SHANE S. (2003), A General Theory of Entrepreneurship. The Individual-Opportunity Nexus, Northampton, MA, Edward Elgar.

STEYAERT C. (2007). "Entrepreneuring'as a conceptual attractor? A review of process theories in 20 years of entrepreneurship studies ». Entrepreneurship and regional development, 19(6), p. 453-477. 